\title{
Cooperation Policies for Efficient In-Network Caching
}

\author{
Liang Wang \\ University of Helsinki \\ liang.wang@cs.helsinki.fi
}

\author{
Suzan Bayhan \\ HIIT, Aalto University \\ bayhan@hiit.fi
}

\author{
Jussi Kangasharju \\ University of Helsinki \\ jakangas@cs.helsinki.fi
}

\begin{abstract}
Caching is a key component of information-centric networking, but most of the work in the area focuses on simple enroute caching with limited cooperation between the caches. In this paper we model cache cooperation under a game theoretical framework and show how cache cooperation policy can allow the system to converge to a Pareto optimal configuration. Our work shows how cooperation impacts network caching performance and how it takes advantage of the structural properties of the underlying network.
\end{abstract}

\section{MOTIVATION}

In-network caching is an indispensable component of information-centric networking (ICN). However, managing a group of networked caches is a big challenge. Most previous work on caching in ICN focuses on simple en-route caching $[1,3,5]$, with limited cooperation among the routers. Lack of cooperation engenders redundancy and degrades efficiency of network storage use. In [5] we showed that cooperation efficiently reduces duplicates and boosts performance, however at the cost of (some) increase in network traffic.

Game theory has been used to analyze distributed caching systems [2]. In [4] caching is modeled as a replication game on graphs, showing the existence of Nash equilibrium on arbitrary topologies. However, the impact of network structure on cooperation has been overlooked. Our work focuses on the relationship between cooperation, topological properties, and caching performance. With an optimization model, we find the Pareto frontier of network caching performance and show the area which a cooperation policy can explore.

\section{SYSTEM MODEL}

We use two caching performance metrics: Byte Hit Rate (BHR) and Footprint Reduction (FPR). Traffic footprint is defined as the product of traffic volume and the distance between content source and client. FPR measures the reduction of traffic footprint. Our focus is an ISP which runs this kind of a in-network caching system. In this context, BHR measures the reduction in traffic going outside the ISP and FPR measures the reduction in traffic within the ISP.

\subsection{Caching as a Game}

Let $\mathcal{G}=(V, E)$ represent the ISP network where $V=\left\{v_{i}\right\}$ is the set of routers with cache capacities $C_{i}$, and $E$ is the links between the routers. We have a content set $\mathcal{O}=\{o\}$, and a demand matrix $W=\left\{w_{i}^{o}\right\}, w_{i}^{o}$ indicates the demand for object $o$ on $v_{i}$. Since a router makes caching decisions locally and they are also affected by the content population in the network, i.e., the contents stored by other routers, we model the caching decision as a non-cooperative game. ${ }^{1}$

In our model, a router $v_{i}$ serves a request for a content item either from local storage, or by retrieving it from another router $v_{j}$ within its r-hop neighborhood if $v_{j}$ stores it, or by retrieving it from the content provider $(\mathrm{CP})$. Let searchable set $\mathcal{N}_{i}^{r}$ be the set of routers within the neighborhood of $r$ hops of $v_{i}$. We refer to $r$ as the search radius.

The searchable set depends on the structure of the network $\mathcal{G}$ and the characteristics of $v_{i}$, e.g., higher degree centrality nodes have a larger set, so we allow each $v_{i}$ to select its own $r$. Due to this heterogeneity, neighborhood relation is not necessarily symmetric. $\mathcal{L}_{i}$ is $v_{i}$ 's cache configuration representing the content set stored locally at $v_{i}$. In the game theory context, $\mathcal{L}_{i}$ is the action of $v_{i}$, and is feasible if both cache capacity and maximum replica constraints are met. The former ensures that a cache cannot store more objects than it has storage capacity and the latter aims to decrease redundancy and favor more efficient cache space usage. We let each item to be stored in at most $N_{\text {copy }}$ routers in the network. However, as $v_{i}$ is aware of the contents only in $\mathcal{N}_{i}^{r}$, we modify the maximum replica constraint for $o$ as follows: $\sum_{v_{j} \in \mathcal{N}_{i}^{r}} 1_{\left[o \in \mathcal{L}_{j}\right]} \leqslant\left\lceil\frac{\left|\mathcal{N}_{i}^{r}\right|}{|V|} N_{\text {copy }}\right]$ where $1_{[x]}$ is the indicator function taking value 1 if boolean expression $x$ is true.

The cache profile of the whole network is the aggregation of all individual cache configurations, $\mathcal{L}=\left(\mathcal{L}_{0}, \mathcal{L}_{1}, \mathcal{L}_{2} \ldots\right)$. $\mathcal{L}_{-i}$ is defined as all other routers except $v_{i}$ sticking to configurations in $\mathcal{L}$. Let $\mathcal{H}_{i}=\bigcup_{\forall v_{j} \in \mathcal{N}_{i}^{r}} \mathcal{L}_{j}$ be the content set that $v_{i}$ can retrieve from its neighborhood, and $\mathcal{R}_{i}=\mathcal{O} \backslash\left(\mathcal{L}_{i} \cup \mathcal{H}_{i}\right)$ be the set of contents that are not stored at $v_{i}$ or in $\mathcal{N}_{i}^{r}$. We assume caching an object locally induces no cost, but retrieving it remotely does and denote the cost by $\alpha_{i}^{o}$. We define $\alpha_{i}^{o}$ as the length of the shortest path between $v_{i}$ and the nearest copy of $o$ it can find in $\mathcal{N}_{i}^{r}$. Total cost on $v_{i}$ under cache configuration $\left(\mathcal{L}_{i}, \mathcal{L}_{-i}\right)$ is:

$$
\operatorname{cost}_{i}\left(\mathcal{L}_{i}, \mathcal{L}_{-i}\right)=\sum_{\forall o \in\left\{\mathcal{H}_{i} \backslash \mathcal{L}_{i}\right\}} w_{i}^{o} \alpha_{i}^{o}+\sum_{\forall o \in \mathcal{R}_{i}} w_{i}^{o} \beta_{i}^{o}
$$

where $\beta_{i}^{o}$ is the length of the shortest path between $v_{i}$ and the $\mathrm{CP}$ that stores $o$. A router gets benefit by serving requests both from its directly attached clients and requests it receives from its neighbor routers. Let us define $\widetilde{\mathcal{N}}_{i}=$ $\left\{v_{j} \mid \forall v_{j} \in V, v_{i} \in \mathcal{N}_{j}^{r}\right\}$ as the set of routers who have $v_{i}$ in

\footnotetext{
${ }^{1}$ Although all routers belong to the same ISP, we do not assume any centralized control for managing the content, i.e., every router makes decisions using only local information.
} 


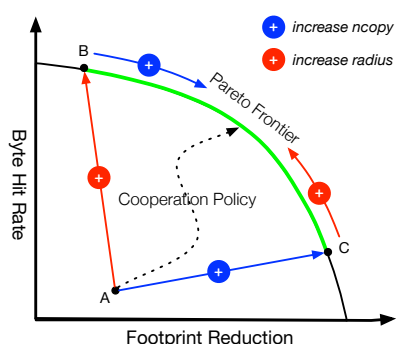

(a) Cooperation policy leads to system Pareto frontier.

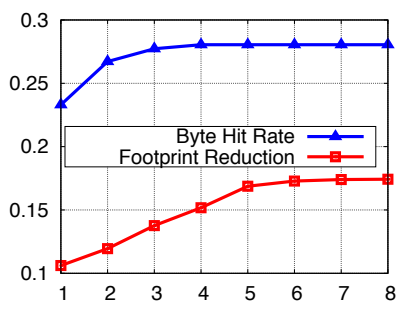

(b) System performance as a function of $N_{\text {copy }}, r=0$

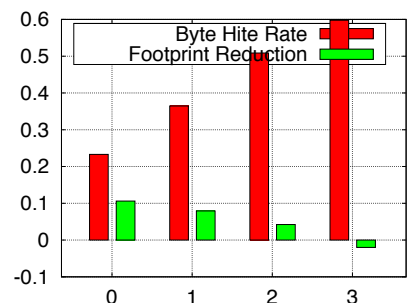

(c) System performance as a function of $r, N_{c o p y}=1$

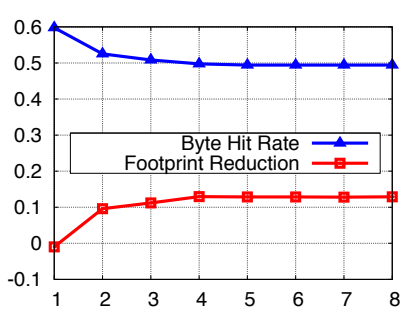

(d) System performance as a function of $N_{\text {copy }}, r=3$

Figure 1: Performance evaluation on a 4-level 2-tree topology. Clients are attached to the leaves.

their searchable sets. The benefit of $v_{i}$ is as follows:

$$
\text { benefit }_{i}\left(\mathcal{L}_{i}, \mathcal{L}_{-i}\right)=\sum_{\forall o \in \mathcal{L}_{i}}\left(w_{i}^{o}+\sum_{\forall v_{j} \in \widetilde{\mathcal{N}_{i}}} w_{j}^{o}\right)
$$

Given $\mathcal{L}_{-i}, v_{i}$ tries to maximize its own payoff which is defined as the difference between benefit and cost, and calculated as follows:

$$
u_{i}\left(\mathcal{L}_{i}, \mathcal{L}_{-i}\right)=\text { benefit }_{i}\left(\mathcal{L}_{i}, \mathcal{L}_{-i}\right)-\operatorname{cost}_{i}\left(\mathcal{L}_{i}, \mathcal{L}_{-i}\right)
$$

subject to the aforementioned feasibility constraints.

A router's best response is given by $\max _{L_{i}} u_{i}\left(\mathcal{L}_{i}, \mathcal{L}_{-i}\right)$ which can be reduced to a knapsack problem with size $C_{i}$, objects $\mathcal{O}$, and object utilities benefit $t_{i}^{o}-$ cost $_{i}^{o}$. However, due to other routers making caching decisions simultaneously, the network state, i.e., cache configurations at each $v_{i}$, may not be stable. Therefore, we need to investigate if the system has a stable point (Nash equilibrium) in which no router has incentive to change its strategy given others stick with $\mathcal{L}_{-i}$. Also, convergence time is of interest.

\subsection{Social Optimal Cache Configuration}

As a router has limited knowledge about the network, its caching decision may lead to sub-optimal performance. To quantify the limits of this sub-optimality, we compute the optimal performance by designing the social optimal cache configuration. We define the social optimal configuration $\mathcal{L}^{\text {opt }}$ as the set of all cache configurations that lead to the network-wide maximum net benefit, and derive it by solving the following optimization problem: $\max \sum_{\forall v_{i} \in V} u_{i}\left(\mathcal{L}_{i}, \mathcal{L}_{-i}\right)$. Using this upper bound, we can calculate the Price of Anarchy (PoA) which shows the performance loss in case of sub-optimal cache configurations.

\section{PRELIMINARY EVALUATION}

Figure 1 shows how $N_{\text {copy }}$ and $r$ impact the performance of caching. Even though the optimization model can calculate the optimal cache profile for given $N_{\text {copy }}$ and $r$ (e.g., point $A$ in Figure 1a), it does not indicate what are the best values for these two parameters. In other words, we can still achieve better performance by tuning $N_{\text {copy }}$ and $r$, because system may be underutilized. However, the optimization model can be used to find Pareto frontier of the performance (green arc $B C$ in Figure 1). When we reach the Pareto frontier, we cannot improve either of BHR or FPR without hurting the other. The fan-shaped area defined by $A B C$ is the area which a cooperation policy can explore to find the best trade-off between the $N_{\text {copy }}$ and $r$. Note that the lines $A B$ and $A C$ are not parallel to the $\mathrm{x}$ - and y-axis in
Figure 1a, since changing either of $r$ or $N_{\text {copy }}$ affects both BHR and FPR, as we show below.

Figures 1b, 1c and 1d show how performance changes along the segments $A B, A C$ and arc $B C$ by increasing $r$ and $N_{\text {copy }}$ respectively. In Figure $1 \mathrm{~b}$, we increase $N_{\text {copy }}$ by fixing $r=0$. We can see both BHR and FPR improving along the line $A C$ in Figure 1a. Since we set $r=0$, this is equivalent to en-route caching, where allowing some duplicates on disjoint paths will clearly improve performance. In Figure 1c, we increase $r$ by fixing $N_{\text {copy }}=1$. As we move from $A$ towards $B$ along the line $A B$ by increasing $r$, we see that BHR increases and FPR drops, even to negative at the extreme, indicating that the search traffic overhead is larger than the traffic reduction gains of caching. The reason is that as search radius increases, a node's neighborhood grows which will increase BHR significantly, but since we allow only one copy in the network, the request needs to be redirected to other nodes which degrades FPR. Figure 1d shows how performance changes along the Pareto frontier after we reach point $B$.

\section{FUTURE DIRECTIONS}

In the future work, we first explore the possibility of discovering a Nash equilibrium of our game model, and find out how close the performance in the steady state is when compared to the Pareto frontier by calculating the Price of Anarchy. We also plan to study how the topological structures impact the cooperation policy and performance. (e.g. how hetero-radius balances between performance gain and search cost based on the router's centrality, degree and etc.) These results can be used to design better distributed heuristics for making caching decisions, while taking fully into account the topological properties of the underlying network.

\section{REFERENCES}

[1] K. Cho, M. Lee, K. Park, T. Kwon, Y. Choi, and S. Pack. Wave: Popularity-based and collaborative in-network caching for content-oriented networks. In Infocom workshop on Name-Oriented Networking, pages 316-321, 2012.

[2] B.-G. Chun, K. Chaudhuri, H. Wee, M. Barreno, C. H. Papadimitriou, and J. Kubiatowicz. Selfish caching in distributed systems: a game-theoretic analysis. In Proceedings of ACM PODC, 2004.

[3] V. Jacobson, D. K. Smetters, J. D. Thornton, M. F. Plass, N. H. Briggs, and R. L. Braynard. Networking named content. In Proceedings of ACM CoNEXT, 2009.

[4] V. Pacifici and G. Dán. Selfish content replication on graphs. In Proceedings of International Teletraffic Congress, 2011.

[5] W. Wong, L. Wang, and J. Kangasharju. Neighborhood search and admission control in cooperative caching networks. In Proceedings of IEEE Globecom, 2012. 